\title{
Local dynamic mechanical properties in model free-standing polymer thin films
}

\author{
Kenji Yoshimoto, Tushar S. Jain, Paul F. Nealey, and Juan J. de Pablo a) \\ Department of Chemical and Biological Engineering, University of Wisconsin-Madison, Madison, \\ Wisconsin 53706-1691
}

(Received 15 December 2004; accepted 25 January 2005; published online 14 April 2005)

\begin{abstract}
High-frequency sinusoidal oscillations of a coarse-grained polymer model are used to calculate the local dynamic mechanical properties (DMPs) of free-standing polymer thin films. The storage modulus $G^{\prime}$ and loss modulus $G^{\prime \prime}$ are examined as a function of position normal to the free surfaces. It is found that mechanically soft layers arise near the free surfaces of glassy thin films, and that their thickness becomes comparable to the entire film thickness as the temperature approaches the glass transition $T_{g}$. As a result, the overall stiffness of glassy thin films decreases with film thickness. It is also shown that two regions coexist in thin films just at the bulk $T_{g}$; a melt-like region $\left(G^{\prime}\right.$ $\left.<G^{\prime \prime}\right)$ near the free surfaces and a glass-like region $\left(G^{\prime}>G^{\prime \prime}\right)$ in the middle of the film. Our findings on the existence of a heterogeneous distribution of DMPs in free-standing polymer thin films provide insights into recent experimental measurements of the mechanical properties of glassy polymer thin films. () 2005 American Institute of Physics. [DOI: 10.1063/1.1873732]
\end{abstract}

\section{INTRODUCTION}

The thermal and mechanical properties of polymeric nanostructures are of critical importance in a wide variety of technological applications. In advanced lithography, the stiffness of photoresist patterns is an important parameter in the capillary force induced collapse that arises as rinsing solvents evaporate. ${ }^{1,2}$ The glass transition temperature $T_{g}$ of chemically amplified photoresist thin films is also a key parameter for optimization of lithographic processes. ${ }^{3}$

A number of experimental ${ }^{4-7}$ and theoretical ${ }^{8,9}$ studies have shown that $T_{g}$ of thin polymer films can change with thickness $h$. Mattsson, Forrest, and Börjesson, for example, report that the $T_{g}$ of free-standing polystyrene (PS) thin films decreases monotonically below thicknesses of $100 \mathrm{~nm} .{ }^{4} \mathrm{In}$ contrast, past work by our group suggests that the $T_{g}$ of supported PS thin films can be either larger or lower than the bulk $T_{g}$ for $h<100 \mathrm{~nm}$, depending on the chemical interactions between the substrate and the polymer matrix. ${ }^{5,7}$

Torres, Nealey, and de Pablo have demonstrated in molecular dynamic simulations that the diffusivity of polymer segments is highly heterogeneous in polymer thin films and that it is strongly correlated with deviations of $T_{g}$ from the bulk. ${ }^{8}$ Ellison and Torkelson have recently quantified dynamic heterogeneities in supported PS thin films by using a fluorescence-multilayer technique, which is capable of measuring local $T_{g}$ at different locations in the film. ${ }^{6}$ For relatively thick films $(h=270 \mathrm{~nm})$, the $T_{g}$ near the free surface is shown to be $\approx 30 \mathrm{~K}$ lower than the bulk $T_{g}(=374 \mathrm{~K})$, whereas no change of $T_{g}$ is found in the the middle or near the bottom of the film. For thinner films $(h=24,36 \mathrm{~nm})$, however, a decrease of the local $T_{g}$ is observed across the films, but its magnitude becomes smaller, i.e., 4-14 K. These

${ }^{a)}$ Electronic mail: depablo@engr.wisc.edu results suggest that a mobile layer exists near the free surface, and that a substrate can have a strong influence in the dynamics of polymer thin films.

It is intuitively attractive to anticipate a correlation between a localized, enhanced polymer motion and a local mechanical weakening of a material. To the best of our knowledge, however, the local mechanical properties of polymer thin films have not been measured as a function of position within the film. It is also important to note that the mechanical deformation of polymeric nanostructures observed in advanced lithography ${ }^{1,2}$ takes place at room temperature, i.e., $20 \%-30 \%$ below the $T_{g}$ of amorphous polymers such as PS and poly(methyl methacrylate) (PMMA). The mechanical properties of amorphous polymers are nearly independent of temperature in the glass region, but they change significantly as $T_{g}$ is approached. ${ }^{10,11}$ The extent of softening in glassy thin films will therefore depend not only on thickness but also on the proximity to $T_{g}$.

Recent experimental studies suggest that the overall stiffness of glassy polymer thin films remains comparable to that of the bulk for film thicknesses in the range 30-40 nm. ${ }^{12,13}$ The Young's modulus $E$ of PS thin films, for example, is comparable to that of the bulk for $h>30 \mathrm{~nm} .^{12}$ Also, the propagation of acoustic waves through PMMA thin films exhibits the same velocity as in the bulk for $h$ $>40 \mathrm{~nm}^{13}$ Similar observations emerge from molecular simulations of polymer nanostructures. ${ }^{14,15}$ The overall bending modulus of the glassy nanostructures remains bulk-like until a critical dimension (e.g., line width) approaches $20 \sigma$. The symbol $\sigma$ represents the diameter of the coarse-grained polymer segments in simulations. A length of $20 \sigma$ would translate into 30-40 nm for amorphous PS or PMMA films. ${ }^{16}$

Experiments and simulations therefore suggest that the onset of changes of mechanical properties does not occur until thicknesses on the order of $30-40 \mathrm{~nm}$ are attained. It 
should be noted, however, that most available mechanical measurements have been performed on supported films; it is unclear whether the absence of any changes of the mechanical properties is indicative of a homogeneous distribution of the bulk stiffness, or of a cancellation of the softened free surface with the stiffened layers near the substrate.

In this work, we present results of nonequilibrium molecular dynamics (NEMD) simulations using a coarsegrained polymer model. The local dynamic mechanical properties (DMPs) of free-standing polymer thin films are reported as a function of thickness and temperature. By limiting our study to free-standing films, we are able to eliminate any convolution of effects arising from the presence of a free surface and a substrate. Dynamic properties, such as $G^{\prime}$ and $G^{\prime \prime}$, are given special attention in this work. Previous studies have already examined the purely elastic response of amorphous polymer glasses well below $T_{g},{ }^{14,15}$ near $T_{g}$, however, the loss component of the modulus becomes significant. More importantly, near a free surface, where segmental mobility can be highly enhanced, it is of interest to consider viscous losses, as the local $T_{g}$ might be much smaller than that of the bulk.

We begin our discussion by describing the model and methods employed in this work. We then report results for the local viscoelastic properties, which show how thin films become mechanically and dynamically heterogeneous in free-standing geometries. We also discuss the formation of soft layers near free surfaces, the effect of these soft surface layers on the overall stiffness of glassy thin films, and the coexistence of melt-like regions and glass-like regions in thin films near the glass transition temperature.

\section{SIMULATION MODEL AND METHOD}

\section{A. Model}

We have adopted a coarse-grained polymer model that has been used in previous studies of glasses. ${ }^{9}$ Each polymer chain consists of 32 interactive sites (i.e., monomer particles). The interaction energy function $u(r)$ between nonbonded monomers is given by a shifted Lennard-Jones (LJ) potential, ${ }^{17}$

$$
u(r)= \begin{cases}v(r)-v\left(r_{c}\right)-\left[\frac{d v(r)}{d r}\right]_{r=r_{c}}\left(r-r_{c}\right) & r \leqslant r_{c}, \\ 0 & r>r_{c}\end{cases}
$$

where $r$ is the distance between the two monomers and $r_{c}$ is the cutoff distance $(=2.5 \sigma)$. The function $v(r)$ is a simple Lennard-Jones potential energy function,

$$
v(r)=4 \epsilon\left[(\sigma / r)^{12}-(\sigma / r)^{6}\right],
$$

where $\sigma$ and $\epsilon$ represent the monomer diameter and the interaction energy parameter, respectively. For bonded pairs, $u(r)$ is represented by a simple harmonic potential,

$$
u(r)=k\left(r-r_{0}\right)^{2} / 2,
$$

where $k$ and $r_{0}$ are the spring constant $\left(=2000 \epsilon / \sigma^{2}\right)$ and the bond length $(=1 \sigma)$, respectively. Note that $r_{0}$ is slightly smaller than the distance at the energy minimum of the

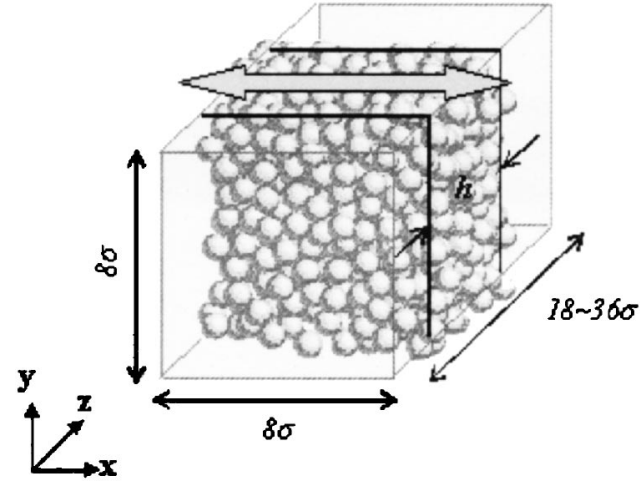

FIG. 1. Schematic view of model free-standing polymer thin film. The arrow on top indicates the oscillatory shearing direction. Each sphere represents the monomer segment.

shifted LJ potential in Eq. (1). Such a choice of parameters inhibits crystallization and facilitates the formation of an amorphous material. ${ }^{9}$ In what follows, all quantities are reported in reduced units, ${ }^{17}$ e.g., distance $r^{*}=r / \sigma$, number density $\rho^{*}=\rho \sigma^{3}$, temperature $T^{*}=k_{B} T / \epsilon$, where $k_{B}$ is the Boltzmann constant, and stress $\tau^{*}=\tau \sigma^{3} / \epsilon$.

The bulk system contains 22 polymer chains under periodic boundary conditions in all three directions. The films consist of 22 or 44 chains, depending on thickness. Figure 1 provides a schematic representation of our thin film systems. Periodic boundary conditions are implemented in the $x$ and $y$ directions; the film thickness $h$ is defined in the $z$ direction.

All systems (bulk and thin films) are first equilibrated at temperatures well above $T_{g}\left(T^{*}=0.60\right)$, and then cooled gradually to a temperature well below $T_{g}\left(T^{*}=0.10\right)$. In all simulation runs, the time step was set at 0.001 in reduced units. For the bulk system, the pressure was fixed at zero and the volume of the system was allowed to fluctuate. For thin films, the box length in the $x$ and $y$ directions was fixed at $8 \sigma$ (see Fig. 1) and the pressure normal to the free surfaces was maintained at zero. The same cooling procedure was repeated with six to ten statistically independent configurations prepared at high temperatures $\left(T^{*}=0.60\right)$. The resulting energy, density, and structure factor at each temperature were found to be the same (within statistical errors) for all simulation runs. By plotting the energy (or the density) as a function of temperature, $T_{g}^{*}$ was estimated to be 0.37 for the thinner films of 22 chains and 0.40 for the bulk and the thicker films of 44 chains. $^{9}$

\section{B. Method}

To calculate the loss and storage modulus of our systems, we implemented a SLLOD algorithm with LeeEdwards periodic boundary conditions. ${ }^{17-20}$ NEMD simulations were performed under an oscillatory shear flow. ${ }^{17,18}$ The temperature was kept constant via a Nose-Hoover chain thermostat. $^{19,20}$ A double thermostat of unit mass was employed. The time-dependent shear strain imparted to our systems, $\gamma^{x y}(t)$, is given by a simple sinusoid of the form 


$$
\gamma^{x y}(t)=\gamma_{\max } \sin \omega t
$$

where $\gamma_{\max }$ and $\omega$ are the amplitude and the frequency of an oscillation, respectively. Superscript $x y$ denotes the two Cartesian components parallel and normal to the shearing direction (see Fig. 1). The shear stress corresponding to $\gamma^{x y}(t)$ can be calculated from the following expression: ${ }^{17}$

$$
\begin{aligned}
\tau^{x y}(t)= & \frac{-1}{V}\left[\sum_{i=1}^{N} m_{i} v_{i}^{x}(t) v_{i}^{y}(t)\right. \\
& \left.-\left.\sum_{i>j} \frac{d U(r)}{d r}\right|_{r=r_{i j}(t)} \frac{r_{i j}^{x}(t) r_{i j}^{y}(t)}{r_{i j}(t)}\right],
\end{aligned}
$$

where $V$ is the volume of the system and $N$ is the total number of monomers. The mass and the $x$ (or $y$ ) velocity component of monomer $i$ are denoted by $m_{i}(=1)$ and $v_{i}^{x}\left(\right.$ or $\left.v_{i}^{y}\right)$, respectively. In the same manner, the magnitude and the $x$ (or $y$ ) component of the vector which connects the positions of monomers $i$ and $j$ are denoted by $r_{i j}$ and $r_{i j}^{x}$ (or $r_{i j}^{y}$ ), respectively. The summation runs over all possible monomer pairs.

In a homogeneous bulk system, the local stresses at any spatial point are expected to be the same as that obtained from Eq. (5). In thin films, however, the local stresses depend strongly on position (e.g., near the free surfaces or at the middle of the film) and direction (e.g., tangential or normal to the free surfaces). ${ }^{21}$ To consider such a heterogeneous stress distribution explicitly, we have applied a onedimensional version of the Irving-Kirkwood stress tensor: ${ }^{21}$

$$
\begin{aligned}
\tau^{x y}(t, z)= & \frac{-1}{A}\left\{\sum_{i=1}^{N} m_{i} v_{i}^{x}(t) v_{i}^{y}(t) \delta\left[z-r_{i}^{z}(t)\right]\right. \\
& -\left.\sum_{i>j} \frac{d U(r)}{d r}\right|_{r=r_{i j}(t)} \frac{r_{i j}^{x}(t) r_{i j}^{y}(t)}{r_{i j}(t)} \\
& \left.\times \frac{1}{\left|r_{i j}^{z}(t)\right|} \Theta\left[\frac{z-r_{i}^{z}(t)}{r_{i j}^{z}(t)}\right] \Theta\left[\frac{r_{j}^{z}(t)-z}{r_{i j}^{z}(t)}\right]\right\},
\end{aligned}
$$

where $\Theta$ is the Heaviside step function. Note that $\tau^{x y}(t, z)$, represents the average local stress over the area of the $x y$ plane, denoted by $A$. The kinetic term in Eq. (6) and the local density,

$$
\rho(t, z)=\frac{1}{A} \sum_{i=1}^{N} m_{i} \delta\left[z-r_{i}^{z}(t)\right]
$$

were estimated with a technique developed by Daivis, Travis, and Todd. ${ }^{22}$ The two velocity components, $v_{i}^{x}$ and $v_{i}^{y}$ are independent, so the kinetic terms in Eqs. (5) and (6) become approximately zero.

Over 50 oscillation cycles were applied to all systems. In each cycle, instantaneous stresses were determined from Eq. (5) and (6) at regular time intervals. The dominant frequency of the stress data was analyzed by a discrete Fourier transform. As expected, all results were consistent with the frequency of the applied strain, namely, $\omega$ in Eq. (4). The stress data were subsequently fitted to an expression of the form: ${ }^{10}$
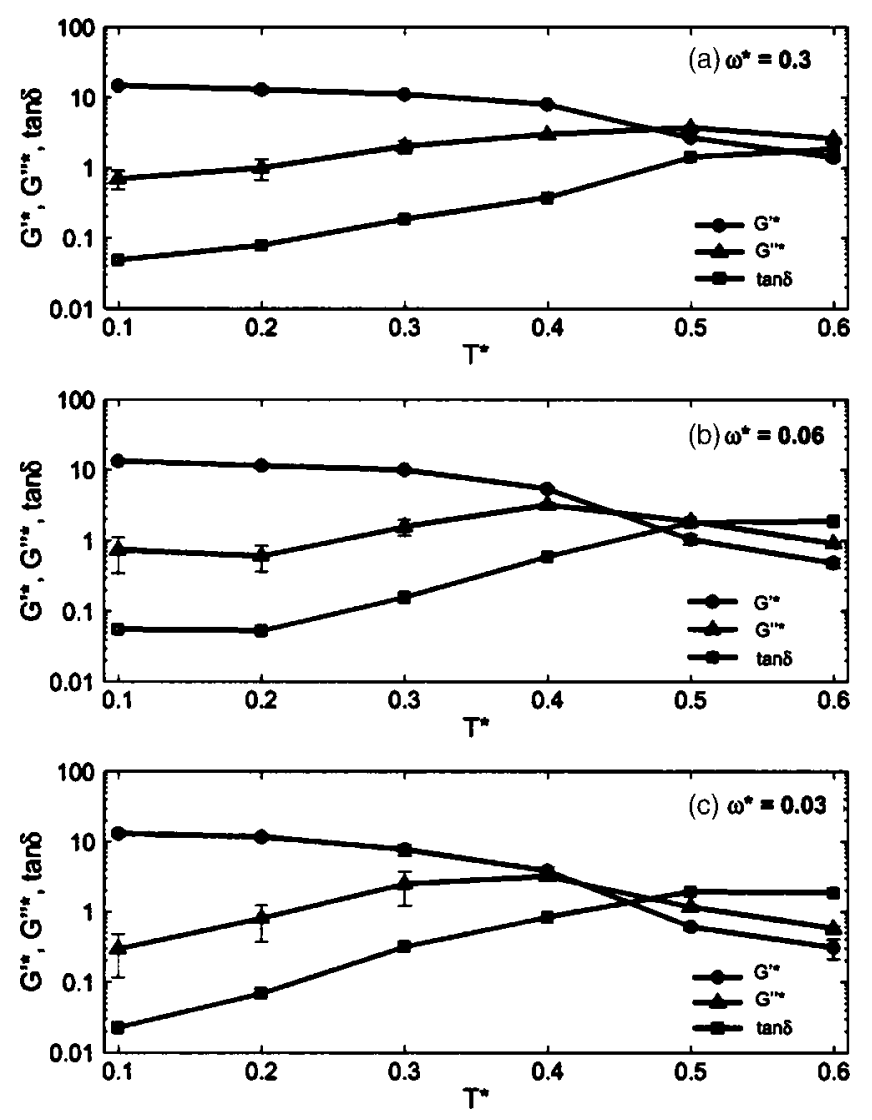

FIG. 2. DMPs of the bulk at (a) $\omega^{*}=0.3$, (b) $\omega^{*}=0.06$, (c) $\omega^{*}=0.03$ vs temperature. In each figure, $G^{\prime *}, G^{\prime \prime *}$, and $\tan \delta$ are shown in $\bigcirc, \square$, and $\triangle$, respectively.

$$
\left\{\begin{array}{l}
\tau^{x y}(t) / \gamma_{\max }=G^{\prime}(\omega) \sin \omega t+G^{\prime \prime}(\omega) \cos \omega t \\
\tau^{x y}(t, z) / \gamma_{\max }=G^{\prime}(\omega, z) \sin \omega t+G^{\prime \prime}(\omega, z) \cos \omega t
\end{array} .\right.
$$

In the fitting, the sum of the squared differences between the calculated stress and that predicted from Eq. (8) was minimized by optimizing the two independent parameters, $G^{\prime}(\omega)$ and $G^{\prime \prime}(\omega)$ for the bulk, or $G^{\prime}(\omega, z)$ and $G^{\prime \prime}(\omega, z)$ for thin films. Note that $G^{\prime}$ and $G^{\prime \prime}$ must be independent of the strain amplitude $\gamma_{\max }$ at a given frequency as shown in Eq. (8). We verified this independence by performing the same calculations described above with several different amplitudes. In what follows, we show the DMPs averaged over different initial configurations and/or different strain amplitudes.

\section{RESULTS AND DISCUSSION}

\section{A. DMPs for the bulk}

In Fig. 2, the DMPs for the bulk are shown as a function of temperature. The frequencies $\omega^{*}$ employed in this study are $0.30,0.06$, and 0.03 . We should note that, in order to obtain the DMPs at $\omega^{*}=0.03$, we have run the NEMD simulations over $10^{8}$ time steps; this required one month of computer time on a $2 \mathrm{GHz}$ CPU.

At $\omega^{*}=0.3$ [Fig. 2(a)], it is observed that the decrease of the stiffness $G^{\prime *}$ as the glass transition is traversed is only a factor of 10 . The loss modulus $G^{\prime \prime^{*}}$ does not exhibit a clear peak in the vicinity of the glass transition. In addition, the intersection point of $G^{\prime *}$ and $G^{\prime \prime^{*}}$, which can be viewed as 

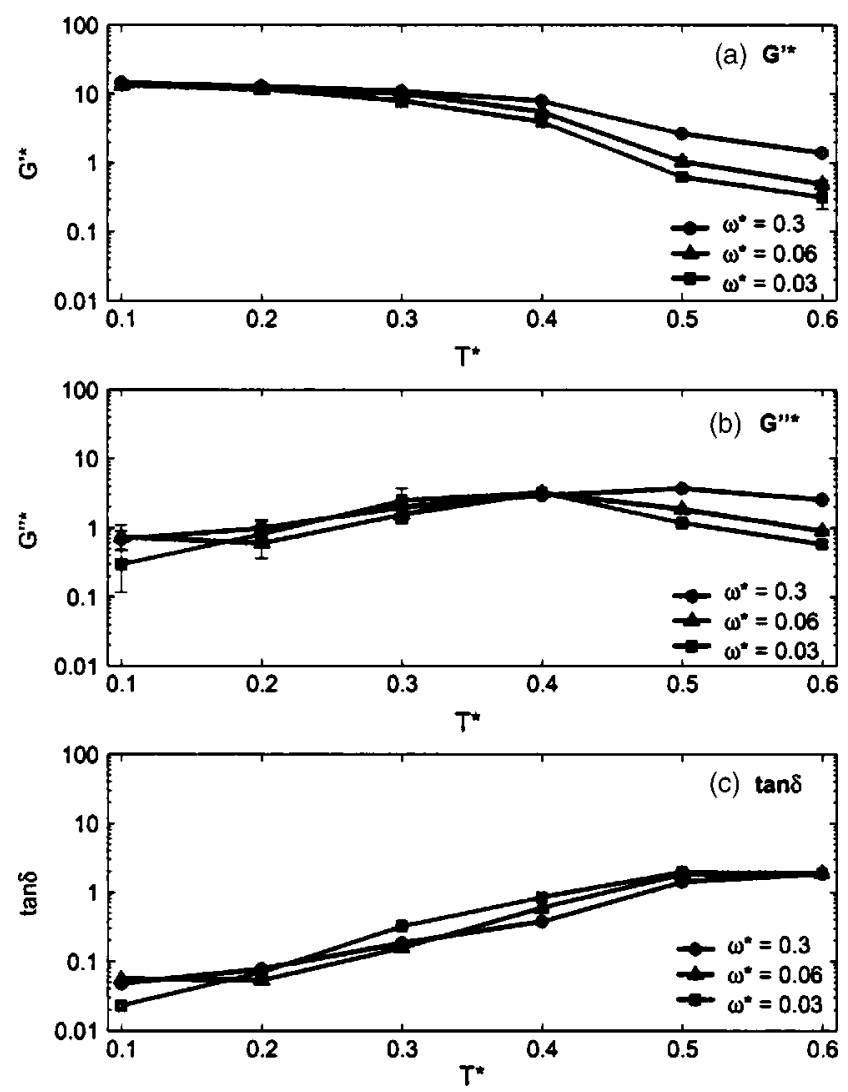

FIG. 3. DMPs of the bulk at different frequencies vs temperature; (a) $G^{\prime *}$ vs $T^{*}$, (b) $G^{\prime \prime^{*}}$ vs $T^{*}$, (c) $\tan \delta$ vs $T^{*}$. In each figure, $\bigcirc, \square$, and $\triangle$ represent the data obtained at $\omega^{*}=0.3,0.06$, and 0.03 , respectively.

another definition of $T_{g}^{*}$, is $\approx 20 \%$ larger than the one estimated from the density-temperature curve in the cooling procedure $(=0.40)$. As the frequency is decreased [Figs. 2(b) and 2(c)], however, the glass transition becomes more pronounced, as evidenced by a larger decrease of $G^{\prime *}$ and a sharper appearance of the peak in $G^{\prime \prime *}$. The intersection of $G^{\prime *}$ and $G^{\prime \prime *}$ also moves towards $T^{*}=0.40$ [see Figs. 2(a) and 2(c)].

To clarify the DMPs dependence of frequency, each property in Fig. 2 is combined into one figure (Fig. 3). Far below $T_{g}^{*}$, it is observed that $G^{\prime *}$ is nearly constant [Fig. 3(a)] whereas $G^{\prime \prime *}$ decreases with $\omega^{*}$ [Fig. 3(b)]. Consequently, $\tan \delta$ becomes smaller at lower frequencies [Fig. 3(c)] and the system becomes more elastic. On the other hand, above $T_{g}^{*}$, both $G^{\prime *}$ and $G^{\prime \prime *}$ decrease with $\omega^{*}$ by the same magnitude, and therefore $\tan \delta$ remains nearly constant; our bulk systems are clearly viscoelastic in the melt regime. Note that the $\alpha$ peak is not observable in Fig. 3(c). (Since the $\alpha$ peak is broadened at higher frequencies, the highest temperature considered here is not large enough to see a clear peak.) Unfortunately, our calculations become inaccurate for the melt at higher temperatures $(>0.6)$; the stiffness is too small to generate a detectable stress response.

\section{B. Local DMPs for thin films}

For thin films, we focus our attention on results at the smallest frequency, namely, $\omega^{*}=0.03$. Figure 4 shows the local $G^{\prime *}, G^{\prime \prime *}$, and density as a function of $z^{*}$ (see Fig. 1) at

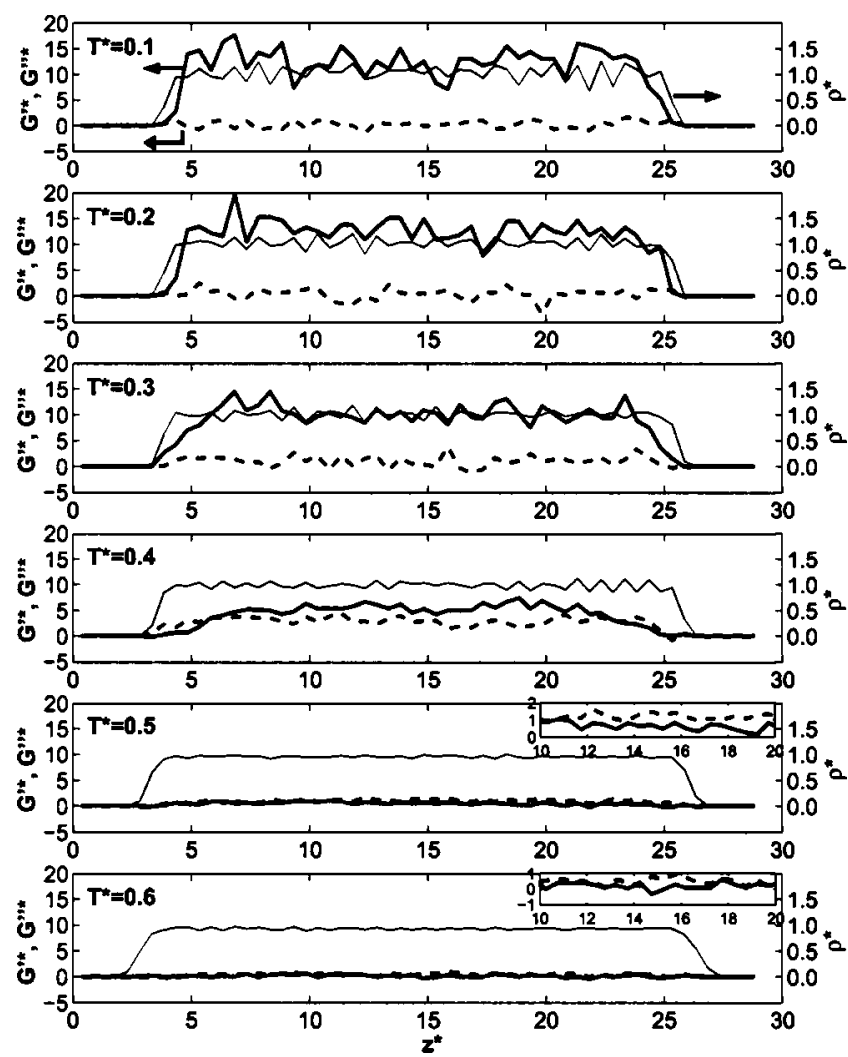

FIG. 4. Local DMPs of thicker films $\left(h^{*} \approx 22 \sigma\right)$ at $\omega^{*}=0.03$ as a function of the $z^{*}$ coordinate: $G^{\prime *}\left(z^{*}\right)$ in bold solid line, $G^{\prime \prime *}\left(z^{*}\right)$ in bold dashed line, and $\rho^{*}\left(z^{*}\right)$ in thin solid line. The scale on the left axis is for $G^{\prime *}\left(z^{*}\right)$ and $G^{\prime \prime^{*}}\left(z^{*}\right)$, and that on the right axis is for $\rho^{*}\left(z^{*}\right)$. From the top to the bottom figure, temperature is increased constantly by 0.1 in reduced units. The insets at $T^{*}=0.05$ and 0.6 show local DMPs near the middle of film.

different temperatures. The left and right edges of $G^{\prime *}\left(z^{*}\right)$, $G^{\prime \prime *}\left(z^{*}\right)$, and $\rho^{*}\left(z^{*}\right)$ are zero beyond the polymer-vacuum interface. The film thickness $h^{*}$ is defined as the distance between the two points of $z^{*}$ at which $\rho^{*}\left(z^{*}\right)$ is equal to half of the bulk density. For the case of Fig. $4, h^{*}$ is $\approx 21-22$ depending on the temperature.

Far below the bulk $T_{g}^{*}$ (i.e., $\left.T^{*}=0.1,0.2\right), G^{\prime *}\left(z^{*}\right)$ exhibits a sharp increase near the free surfaces and a fluctuation around a bulk value in the middle of the film. In contrast, $G^{\prime \prime}\left(z^{*}\right)$ is vanishingly small over the entire film thickness. Most of the film (except the immediate vicinity of the free surfaces) may be assumed rigid and elastic. As the temperature approaches the bulk $T_{g}^{*}$ (i.e., $T^{*}=0.3,0.4$ ), however, $G^{\prime *}\left(z^{*}\right)$ rises more gently near the free surfaces and attains the bulk value deep inside the film. In addition, the magnitude of $G^{\prime *}\left(z^{*}\right)$ becomes comparable to that of $G^{\prime \prime *}\left(z^{*}\right)$ in the middle of the film. These results reveal a gradual transition of the thin film from the glass to the melt. Note that, just at the bulk $T_{g}^{*}$ (i.e., $T^{*}=0.4$ ), a small portion of $G^{\prime \prime^{*}}\left(z^{*}\right)$ becomes larger than $G^{\prime *}\left(z^{*}\right)$ near the free surfaces, i.e., a phenomenon akin to "surface melting" 6 occurs. Meanwhile, a glass-like region $\left[G^{\prime *}\left(z^{*}\right)>G^{\prime \prime *}\left(z^{*}\right)\right]$ is still maintained in the middle of the film. This finding is consistent with the experimental observations on PS thin films mentioned above; a smaller $T_{g}$ at the free surface, ${ }^{6,23}$ but no change in $T_{g}$ deep inside the film. ${ }^{6}$ By further increasing the temperature above the bulk $T_{g}^{*}$ (i.e., 


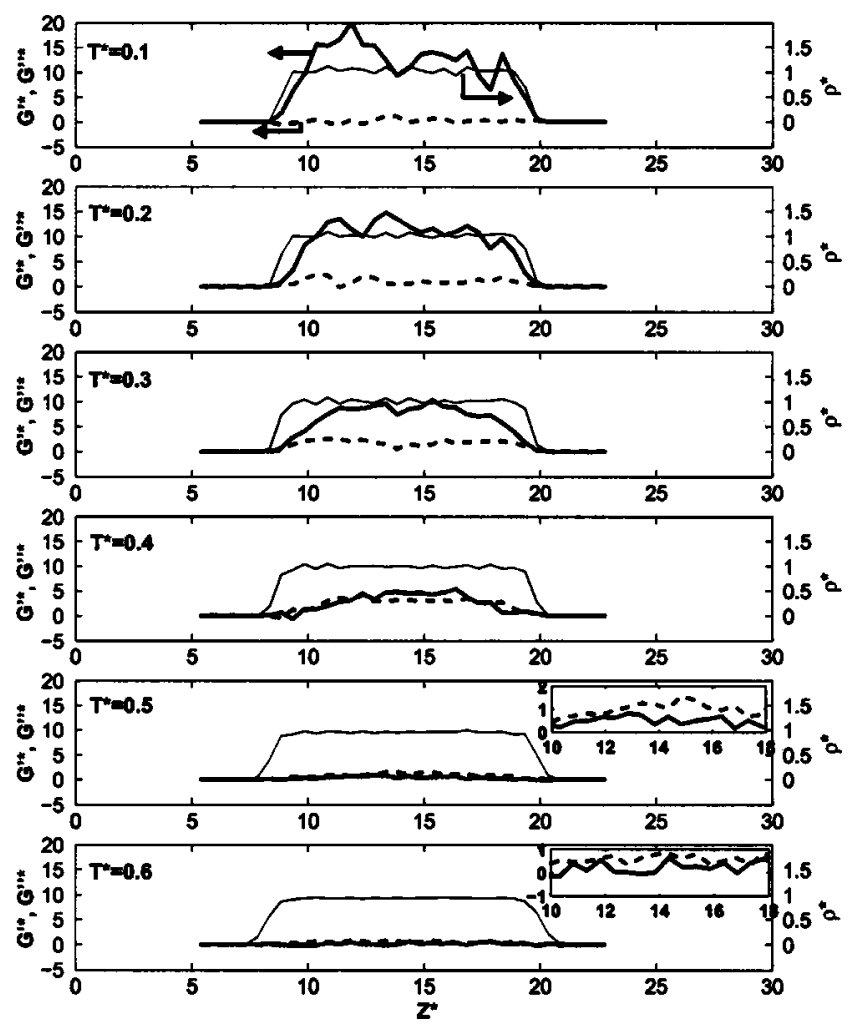

FIG. 5. Local DMPs of thinner films $\left(h^{*} \approx 11 \sigma\right)$ at $\omega^{*}=0.03$ as a function of $z^{*}$ coordinate. The symbols and scales are the same as those in Fig. 4.

$\left.T^{*}=0.5,0.6\right), G^{\prime \prime *}\left(z^{*}\right)$ becomes larger than $G^{\prime *}\left(z^{*}\right)$ for most values of $z^{*}$; the entire film turns into a highly viscoelastic melt.

It is also interesting to observe in Fig. 4 that the heterogeneous behavior of $G^{\prime *}\left(z^{*}\right)$ and $G^{\prime \prime *}\left(z^{*}\right)$ does not simply correspond to the local density profile, particularly near the free surfaces. Far below the bulk $T_{g}^{*}$, there exists a small lag between $G^{\prime *}\left(z^{*}\right)$ and $\rho^{*}\left(z^{*}\right) ; \rho^{*}\left(z^{*}\right)$ reaches the bulk density first, and then $G^{\prime *}\left(z^{*}\right)$ rises to the bulk $G^{\prime *}$. This delay of $G^{\prime *}\left(z^{*}\right)$ is mainly due to the lower density near the free surfaces. Note that the transition of $\rho^{*}\left(z^{*}\right)$ from zero to a bulk density is completed within $1-2 \sigma$ at all temperatures, and the shoulder slope of $\rho^{*}\left(z^{*}\right)$ is only weakly dependent on temperature. Meanwhile, the shoulder slope of $G^{\prime *}\left(z^{*}\right)$ becomes milder at higher temperatures (i.e., $T^{*}=0.3,0.4$ ), and several of the surface underlying layers exhibit a $G^{\prime *}\left(z^{*}\right)$ below the bulk value, even if a bulk density has been attained (see, for example, $z^{*}=4.0-6.0$ and $z^{*}=25.5-24.0$ at $T^{*}=0.3, z^{*}$ $=3.5-6.5$, and $z^{*}=25.0-22.0$ at $\left.T^{*}=0.4\right)$. This decrease of the stiffness at bulk-like densities is consistent with observations that the motion of polymer segments near interfaces is highly cooperative, ${ }^{9}$ and that the activation energy for segmental motions near free surfaces is much lower. ${ }^{24}$ Faster segmental motions near the free surfaces can initiate a global rotational motion that includes many segments, ${ }^{9}$ and the mobility of polymer segments in the film can be enhanced even under bulk-like conditions. ${ }^{24}$ Above the bulk $T_{g}^{*}$, we could not find a clear relationship between $\rho^{*}\left(z^{*}\right)$ and $G^{\prime *}\left(z^{*}\right)$ or between $\rho^{*}\left(z^{*}\right)$ and $G^{\prime \prime}\left(z^{*}\right)$, mainly as a result of relatively large fluctuations.

The behavior of $11 \sigma$ films (see Fig. 5) is similar to that
TABLE I. Ratio of the soft surface layers $h^{*}$ to the entire film thickness $h^{*}$.

\begin{tabular}{cccc}
\hline \hline$T^{*}$ & $h_{s}^{*}$ & $h^{*}$ & $\left(h_{s}^{*} \times 2\right) / h^{*}$ \\
\hline 0.3 & $2.5-3.0$ & 22 & $0.23-0.27$ \\
& $3.0-3.5$ & 11 & $0.55-0.64$ \\
\hline \hline
\end{tabular}

of the thicker films; we also detect the emergence of a soft surface layer and surface melting. In order to compare the thickness of soft surface layers $h_{s}^{*}$ in Figs. 5 and 4, we define $h_{s}^{*}$ as a surface region for which $G^{\prime *}\left(z^{*}\right)<G_{b u l k}^{\prime *}$. The results at $T^{*}=0.3$ are summarized in Table I. As shown in Table I, $h_{s}^{*}$ for $h^{*}=11$ is found to be between 3.0 and 3.5, approximately the same as $h_{s}^{*}$ for $h^{*}=22$. Consequently, the ratio of the soft surface layers $\left(h_{s}^{*} \times 2\right)$ to the overall film thickness $h^{*}$ for the $11 \sigma$ thick film becomes $55 \%-64 \%$, which is more than double that for the $22 \sigma$ film. With such a large surface ratio, it is clear how significant surface effects become on the mechanical properties of free-standing glassy thin films.

\section{Overall DMPs for thin films}

By integrating $G^{\prime}(z)$ and $G^{\prime \prime}(z)$ over the film thickness, one can also calculate the overall DMPs for free-standing polymer thin films:

$$
G^{\prime}=\frac{1}{h} \int_{h} G^{\prime}(z) d z, \quad G^{\prime \prime}=\frac{1}{h} \int_{h} G^{\prime \prime}(z) d z .
$$

The results are shown in Fig. 6. In the glass region, the $G^{\prime *}$ curve for the $22 \sigma$ film ( $\triangle$ with solid line) almost overlaps with that for the bulk ( $\bigcirc$ with solid line); however, that for the $11 \sigma$ film ( $\square$ with solid line) exhibits a systematic departure from the other two. As discussed in the preceding section, a decrease of $G^{\prime}$ in thinner films is due to the greater contribution (greater surface to volume ratio) of soft surface

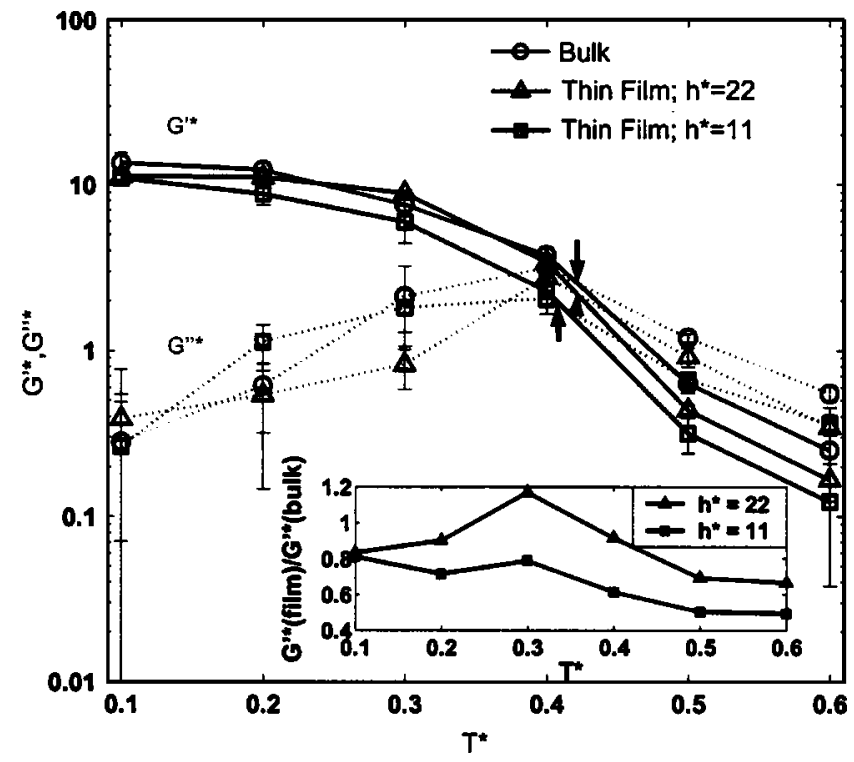

FIG. 6. Overall DMPs of thin films at $\omega^{*}=0.03$ vs temperature; $\bigcirc$ for the bulk, $\triangle$ for $22 \sigma$ thick film, $\square$ for $11 \sigma$ thick film. $G^{\prime *}$ and $G^{\prime \prime *}$ are shown with solid and dashed lines, respectively. An arrow indicates the intersecting point of the two curves of $G^{\prime *}$ and $G^{\prime \prime *}$. The inlet shows the ratio of $G^{\prime *}$ for the thin films to the bulk $G^{\prime *}$ as a function of temperature. 
layers to the overall behavior of the system. The magnitude of the decrease in $G^{\prime}$ is further clarified in the inset of Fig. 6. The $G^{\prime}$ for the $11 \sigma$ film is found to be $60 \%-80 \%$ of the bulk $G^{\prime}$ in the glass region $\left(T^{*}<0.4\right)$. Such a large decrease of stiffness could become a large limiting factor for the semiconductor industry in its attempts to produce sub-50-nm pitched patterns.

\section{CONCLUSIONS}

In our calculations of the local DMPs for free-standing polymer thin films with oscillatory deformations, it is shown that soft layers are formed near the free surfaces of glassy thin films, and the ratio of the soft layers to the entire film thickness becomes larger as the temperature becomes closer to the bulk $T_{g}$ or as the film thickness is decreased. For example, at $T^{*^{\circ}}=0.3$ (i.e., $T / T_{g}=0.75$ ), which is equivalent to room temperature for PS and PMMA, we found that the ratio of soft surface layers becomes more than $50 \%$ of the entire film thickness and $G^{\prime}$ becomes $\approx 70 \%$ of the bulk $G^{\prime}$ for $11 \sigma$ thick films. We are currently working on a similar problem in supported thin films; we anticipate that the free surface softening might be canceled by the interfacial stiffening that can arise near a substrate.

\section{ACKNOWLEDGMENTS}

This work was supported by the Semiconductor Research Corporation (SRC) and the National Science Foundation (NSF) NIRT program.
${ }^{1}$ M. P. Stoykovich et al., Adv. Mater. (Weinheim, Ger.) 15, 1180 (2003).

${ }^{2}$ K. Yoshimoto et al., J. Appl. Phys. 96, 1857 (2004).

${ }^{3}$ L. F. Thompson, C. Willson, and M. J. Bowden, Introduction to Microlithography, 2nd ed. (American Chemical Society, Washington, D. C., 1994).

${ }^{4}$ J. Mattsson, J. A. Forrest, and L. Börjesson, Phys. Rev. E 62, 5187 (2000).

${ }^{5}$ R. S. Tate et al., J. Chem. Phys. 115, 9982 (2001).

${ }^{6}$ C. J. Ellison and J. M. Torkelson, Nat. Mater. 2, 695 (2003).

${ }^{7}$ D. S. Fryer et al., Macromolecules 34, 5627 (2001).

${ }^{8}$ J. A. Torres, P. F. Nealey, and J. J. de Pablo, Phys. Rev. Lett. 85, 3221 (2000).

${ }^{9}$ T. S. Jain and J. J. de Pablo, J. Chem. Phys. 120, 9371 (2004).

${ }^{10}$ R. S. Lakes, Viscoelastic Solids (CRC, Boca Raton, FL, 1998).

${ }^{11}$ D. W. Van Krevelen, Properties of Polymers, 3rd ed. (Elsevier, Amsterdam, Netherlands, 1990).

${ }^{12}$ C. M. Stafford et al., Nat. Mater. 3, 545 (2004).

${ }^{13}$ Y. C. Lee, K. C. Bretz, F. W. Wise, and W. Sachese, Appl. Phys. Lett. 69, 1692 (1996).

${ }^{14}$ T. R. Böhme and J. J. de Pablo, J. Chem. Phys. 116, 9939 (2002).

${ }^{15}$ K. Van Workum and J. J. de Pablo, Nano Lett. 3, 1405 (2003).

${ }^{16}$ M. Rubinstein and R. H. Colby, Polymer Physics (Oxford University Press, New York, 2003).

${ }^{17}$ M. P. Allen and D. J. Tildesley, Computer Simulation of Liquids (Oxford University Press, New York, 1987).

${ }^{18}$ H. Komatsugawa and S. Nosé, Phys. Rev. E 51, 5944 (1995).

${ }^{19}$ C. J. Mundy, J. I. Siepmann, and M. L. Klein, J. Chem. Phys. 103, 10192 (1995).

${ }^{20}$ Z. Xu, J. J. de Pablo, and S. Kim, J. Chem. Phys. 102, 5836 (1995).

${ }^{21}$ F. Varnik, J. Baschnagel, and K. Binder, J. Chem. Phys. 113, 4444 (2000).

${ }^{22}$ P. J. Daivis, K. P. Travis, and B. D. Todd, J. Chem. Phys. 104, 9651 (1996).

${ }^{23}$ N. Satomi, K. Tanaka, A. Takahara, and T. Kajiyama, Macromolecules 34, $6420(2001)$.

${ }^{24}$ T. S. Jain and J. J. de Pablo, Phys. Rev. Lett. 92, 155505 (2004). 